\title{
Icosahedral packing of RNA viral genomes
}

\author{
Joseph Rudnick and Robijn Bruinsma* \\ Department of Physics and Astronomy, UCLA, Box 951547, Los Angeles, CA 90095-1547
}

(Dated: November 15, 2018)

\begin{abstract}
Recent studies reveal that certain viruses package a portion of their genome in a manner that mirrors the icosahedral symmetry of the protein container, or capsid. Graph theoretical constraints forbid exact realization of icosahedral symmetry. This paper proposes a model for the determination of quasi-icosahedral genome structures and discusses the connection between genomic structure and viral assembly kinetics.
\end{abstract}

PACS numbers: 87.15.Nn, 81.16.Dn, 61.50.Ah, 87.16.Dg

An essential step in the assembly of any virus is the structural reorganization of the single stranded (ss) or double stranded (ds) RNA and DNA molecules that comprise the genome into a form that fits inside the viral capsid. This involves a well-known structural incongruity; the protein capsid shells of nearly all sphere-like viruses adopt icosahedral symmetry while, as discussed below, a viral genome of less than twelve segments fundamentally cannot assume an icosahedral conformation in view of its one-dimensional, chain-like primary structure [1]. In fact, microscopy studies of the structure of the ds DNA genome of bacteriophage viruses reveal a completely nonicosahedral spool-like organization 2]. A semi-flexible chain - such as ds DNA - that is confined inside a sphere having an interior surface that repels this chain and a radius comparable or smaller than the chain persistence length will, indeed, adopt such a spool-like structure as its free energy minimum [3, 4] .

The problem of the interior organization of ss RNA viral genomes is more delicate. SS nucleotide chains have a much greater conformational flexibility than duplex chains, so ss RNA chains are better able to adjust to the icosahedral symmetry of the capsid than ds DNA chains. The secondary structure of RNA molecules in solution is characterized by paired segments conntected by branch points, unpaired "bubbles" and "hair pins" (see Fig. [2). The various competing secondary structures can be analyzed by statistical mechanical methods [5]. Moreover, unlike the case of the bacteriophage viruses, for a significant number of ss RNA viruses, capsid assembly requires the presence of the genome molecules [6]. This "co-assembly," which can take place spontaneously by self-assembly [6], is due in part to non-specific electrostatic attraction between RNA molecules and capsid proteins. Attractive interactions between the genome and the interior surface of the icosahedral capsid should promote icosahedral order. X-ray diffraction studies reveal that the outer layer of the genome of a number of ss RNA viruses such as Cowpea Chlorotic Mottle Virus (CCMV) [1, 17, 8], Flock House Virus (FHV) 1, 7, 9], cali-

\footnotetext{
*Instituut-Lorentz/LION, Universiteit Leiden, 2300 RA, Leiden, The Netherlands
}

civirus 1, 7, 10], Cowpea Mosaic Virus (CMV) 1, 7, 11, Turnip Yellow Mosaic Virus (TYMV) 1, 7, 12], indeed adopt at least partial icosahedral symmetry.

A particularly striking illustration of icosahedral ordering is provided by the Nodaviridae group of viruses. The FHV and Pariacoto Nodaviridae viruses have so-called " $\mathrm{T}=3$ " icosahedral capsids, as shown in Fig. 1 The

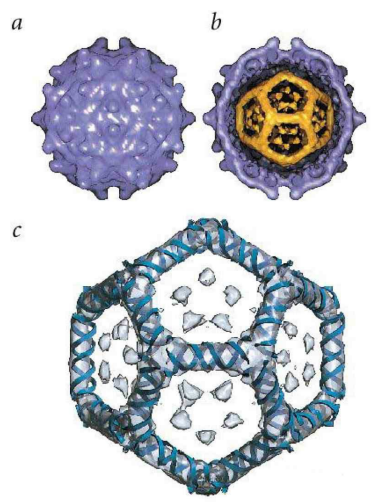

FIG. 1: Image of the pariocoto virus, reconstructed from cryoelctron micrographs by Tang, et. al. 13]

genome of these viruses consists of two single-stranded RNA molecules. One of these two molecules, RNA2, consists of about 1,400 (FHV), respectively 1,300 (Pariacoto) base pairs and encodes for "Protein $\alpha$," a precursor of the capsid protein. This molecule plays a key stabilizing role in capsid assembly [6], and it is probably the part of the genome that is resolved in the diffraction experiments. As shown in Figs. 10 and c, the icosahedral portion of the RNA genome is distributed over a dodecahedral cage formed by the low-curvature borders of twelve five-fold pyramids that together make up the $\mathrm{T}=3$ rhombic tricontahedron capsids of the FHV and Pariacoto viruses. Even though the genome is ss RNA, the portions shown in Fig. 1 actually have the form of double helices with the two strands oriented in the standard way with opposite 3' to 5' directions. These paired double-helical segments have a length per edge of about 10 bases for the FHV structural study and 25 bases for the Pariacoto structural study. Given the 30 edges of a dodecahedron, this means that for FHV about $43 \%$ of RNA2 
contributes to the double-helical sections, and for Pariocoto essentially $100 \%$ of RNA2. If we assume that the 20 (unresolved) vertices are also structurally identical, we would have to connect together the 30 double-helical segments with 20 identical three-strand branch-points (see Fig. 2A) placed at the vertices of the dodecahedron. The resulting structure has true icosahedral symmetry (apart from the different base-pair sequences for the different segments), but it actually consists of 12 separate, interlocking $R N A$ rings, which is inconsistent with the structure of the Nodaviridae genome [14]. This structural conflict is closely connected to a classical result of graph theory, which states that it is impossible to construct a one-dimensional path restricted to the edges of either an icosahedral or a dodecahedral structure while visiting every edge just once [15, 16] (a dodecahedron has the same symmetry properties as an icosahedron). The proposition that the 20 vertices obey icosahedral symmetry is thus incorrect. As shown below, information concerning the structural inhomogeneity of the vertices actually provides us with important information on the formation history of the virus. It is the aim of the present article to present a statistical mechanical model for quasiicosahedral ordering of ss RNA viral genomes.

On the basis of the above-mentioned X-ray structure studies, we set the following constraints on the secondary structure of RNA2:

(i) the edges of the dodecahedron should be occupied by the rigid duplex segments.

(ii) the branch points, bubbles, and hair-pins of the secondary structure should be confined to the vertex regions.

Under these constraints, there are only three possible types of vertex structures, which can be represented diagrammatically as follows. The first vertex type ("A") are pure branch-points, the familiar three-strand junctions of ds RNA and DNA. These vertex types are illustrated in Fig. 2] The arrows in the figure indicate the 3' to 5' direction of the RNA2 molecule. Electrostatic repulsion between the duplex branches of the junction favors $120^{\circ}$ angles between the branches, which have to be deformed by a moderate amount in order to fit on the vertex of a dodecahedron. Cases A1 (a "right-turn" vertex) and A2 (a "left-turn" vertex) are related by a rotation over 180 degrees along one of their branches. The energy cost, $\Delta E_{A}$, is the same for the two cases.

Next, a type "B" vertex consists of a combination bubble/hairpin, as shown in Fig. 2B, which has a total of six variations. Note that the presence of the bubble permits the sharp kink between the two connected duplex sections required at a vertex of the dodecahedron. There are now four RNA strands located at the vertex so the electrostatic energy, $\Delta E_{B}$, of a class $\mathrm{B}$ vertex is presumably higher than that of a class A vertex. Finally, for a class $\mathrm{C}$ vertex, three hair-pin/stem-loops are located at one vertex A class $\mathrm{C}$ vertex requires that six RNA strands
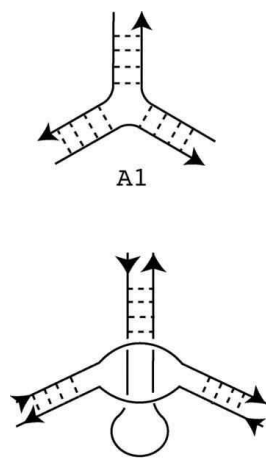

B
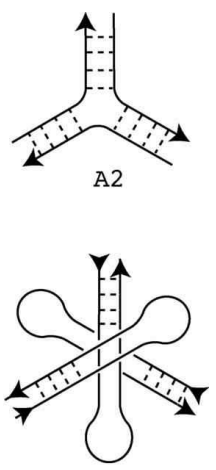

C
FIG. 2: The three types of vertices. In the case of the "A" type vertices (branch points) both a right-turn (A1) and a left-turn (A2) vertex are shown. A type B vertex combines a "bubble" and a "hairpin," while a type C vertex contains only hairpins.

are located at a single vertex and thus has presumably the highest electrostatic energy $\left(\Delta E_{c}\right)$.

The energy cost of a particular secondary RNA2 structure is, then,

$$
H=E^{\mathrm{sol}}+N_{A} \Delta E_{A}+N_{B} \Delta E_{B}+N_{C} \Delta E_{C}
$$

with $E^{\mathrm{sol}}$ the solution energy cost of the secondary structure computed by the existing procedure [5], while $N_{A-C}$ denotes the number of vertices. The non-specific electrostatic binding energy contributes a constant energy that need not be included.

Since, by assumption, Class A vertices have the lowest free energy it is logical to start by constructing RNA configurations that have only A-type junctions, in which case there are no hairpins or stem-loops at all (i.e. $\left.N_{B}=N_{C}=0\right)$. That does not mean that the genome has to be disconnected. Recall that there are two different possible junctions, related by a $180^{\circ}$ rotation. Since we allow for vertex heterogeneity, we can use both junction types and search for a single-connected genome. In order to do this, we represented the dodecahedral cage by a planar graph. A singly-connected RNA molecule must visit every edge of this graph twice, each case in opposite directions, in order to form the double helical segments on the edges. The existence of this type of path is not forbidden by graph theory. The problem of identifying a permissible path for a ss RNA molecule is equivalent to a finding particular choice for the A1 and A2 vertices at every node of the dodecahedron such that they can be connected by a continuous path. We will call this a Modified Euler Path (MEP). In order to identify MEP's, we carried out a computer search in which one of the nodes of the dodecahedron was singled out as the starting (and ending) point of the MEP, while all other vertices are either designated as right turn or left turn vertices. This produced 262,144 different candidates for a MEP. Most of the candidate walks were unsuccessful, because the walker arrived at a point at which the con- 
ditions for the walk were violated. However, in 54,272 of the cases a MEP was generated with a mean success rate of $53 / 256=0.207$. . Two examples of such walks are displayed in Fig. [3] Although the (3D) symmetry of these
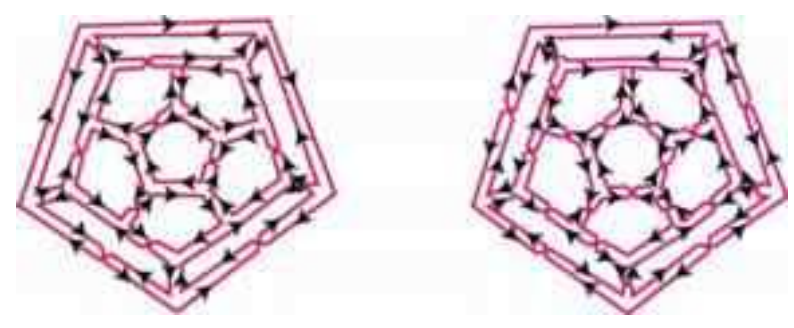

FIG. 3: Two examples of modified Euler paths covering the edges of a dodecahedron. The vertex at which each path begins and ends is in the upper central portion of the dodecahedral graph. The path at the right contains the minimum number of crossings, while the path at the right contains the maximum number of crossings.

RNA structures is very close to icosahedral, it is not exact because the successful MEPs contain a certain number of edges with an extra crossing, as indicated by a cross in Fig. 3. This implies that the double-helical segments that occupy these must have either an extra half turn, or a deficit of one half turn. The MEPs we generated contained a minimum of eleven crossings and a maximum of twenty, corresponding to the two cases shown in Fig. 3. If the energy cost of Type $\mathrm{B}$ and Type $\mathrm{C}$ vertices are significantly higher than that of a Type A vertex, then the pattern with the minimum number of extra crossings should be the lowest free energy structure.

However, it is well known that a path covering a graph with overpasses and underpasses, as shown in Fig. 3] are knotted [17]. Even the MEP that has the minimum number of crossings still is highly knotted. RNA molecules in solution are never knotted (though viral RNA may have "pseudo-knots" [18]), and a knotted RNA molecule released by a virus during infection would not be functional. We thus must exclude knotted RNA structure and hence Class A Quasi-Icosahedral order. Knot-free genomes can be constructed by demanding that, unlike the structures shown in Fig. 3 the secondary structure of RNA molecules inside a virus has the same linearly-branched, circuit-free topology as RNA molecules in solution. When a linearly branched structure decorates a dodecahedral graph, types B and $\mathrm{C}$ vertices unavoidably appear. Given the presumed higher free energy cost of a type $\mathrm{C}$ vertex, we will construct linearly branched genomes with only type A and $B$ vertices. Linearly-branched RNA secondary structures characteristically contains an equal number of branches and hairpin/stemloop structures. That means that there must be an equal number of $A$ and $B$ type vertices, so $N_{A}=N_{B}$. The simplest example of such an A-B structure involves singly-branched RNA molecules, i.e. the molecule consists of a main-chain and a certain number of unbranched side-chains. Secondary structures that are nearly singly-branched are indeed encountered in the spectrum of RNA2, with an energy of about $10 \mathrm{kcal} / \mathrm{mole}$ above the groundstate [18].

To cover the dodecahedral graph with a singlebranched structure, every edge of the dodecahedron must be a link between an A type and a $\mathrm{B}$ type vertex. If this is the case, then the main chain of the RNA molecule must visit every vertex of the dodecahedral graph once and only once. The construction of such a route is a wellknown problem in graph theory, known as a "Hamiltonian Path" 15, 19]. We will focus on the special case of a Hamiltonian Path that starts and ends on the same vertex of a graph, which is a Hamiltonian Cycle, is shown in the lower right-hand corner of Fig. [4 In this case, any point on the graph can be treated as the starting site. To every edge of the graph that is not part of the Hamiltonian Path, we must assign an A-B or a B-A pair of vertices, so for the Hamiltonian Cycle of relevance to the ordering of RNA on an icosahedral virus, we obtain $2^{10}$ different possible configurations. These $2^{10}$ configurations form a subset of the allowed secondary RNA stuctures in solution. For a given base-pair sequence, the optimal secondary structure within this subset can, in principle, be determined by minimizing $H$.

The biological relevance of the secondary structure of the viral genome is, in fact, intimately connected with the assembly scenario of the virus, and this imposes important constraints on the RNA configuration. It has been well-established for ss RNA cylindrical viruses that the RNA molecule acts as a linear growth template 20]. Growth starts from a specific hairpin structure (the "packaging signal") and proceeds by successive addition of protein oligomers under the action of the non-specific protein-RNA interaction. The length of the RNA molecule determines the size of the virus. It is currently not known whether main chain type ss-RNA molecules also can act as growth templates for the icosahedral family of viruses, but the only microscopy study of the growth kinetics of RNA/capsid co-assembly of an icosahedral virus does report that growth starts from a three-protein nucleus followed by the successive addition of oligomer units 21]. The observed co-assembly scenario resmbles the growth of a curved two-dimensional (2D) protein crystal that closes in on itself. The characteristic feature of conventional (slowly) growing crystals is that they are compact. New units are predominantly added to those available sites on the surface of the crystal where the new unit can make a maximum number of bonding contacts with units that are already incorporated. The co-assembly condition requires the presence of RNA material when new units are added. The effect of this process is to even out surface roughness, although with increased growth velocities, the growth surface may roughen.

To examine whether the reported growth scenario is consistent with the proposed Hamiltonian path/cycle model developed above, we assume that the Hamitonian Path construction provides the lowest accessible free en- 
ergy structure as proposed in the previous section. The assembly history for the Hamiltonian Cycle is shown in Fig. 4, in which we show only the main chain part. Edges that are not indicated by heavy lines in the figure below are occupied by a side-chain, ending in a hairpin. Except for the first step, every added pentamer bonds at least to two edges, one occupied by the main chain and one occupied by a side chain. The growth morphology imposed by the Hamiltonian path construction is, then, consistent with compact $2 \mathrm{D}$ growth structures.

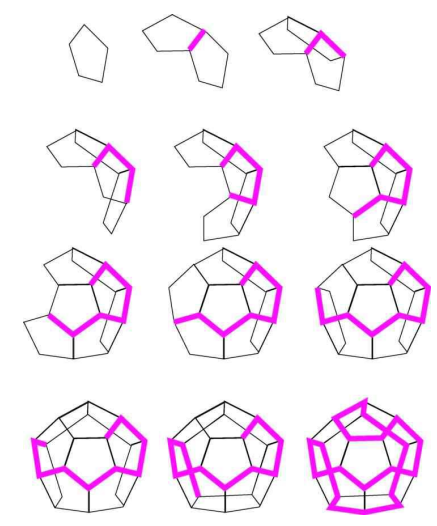

FIG. 4: Assembly of a viral capsid governed by coordinated with the tracing out of a Hamiltonian path by the icosahedrally-ordered RNA. For the fully-assembled capsid, shown in the lower right-hand corner of the figure, all portions of the Hamiltonian path traced out by the RNA main chain are indicated.
In summary, on the basis of elementary graphtheoretical arguments, we propose that the quasiicosahedral organization of unsegmented ss RNA viral genomes is based on the exploitation of the secondary structure of the viral RNA to form a Hamiltonian path or cycle along a subset of the edges of a polyhedron inscribed on the capsid surface, with side-branches covering the remaining edges. The important role for the RNA mainchain structure would be consistent with the main-chain acting as a linear template for the growth of the capsid during the self-assembly process. Our arguments predict that quasi-icosahedral organization should never be encountered for viruses with unsegmented fully duplexed ds DNA or RNA genomes. Experimental tests should be straightforward. For instance, FHV self-assembles under in-vitro conditions. Self-assembly of FHV with a duplexed RNA strand having the same length as the FHV ss RNA should be difficult or impossible. Determining the nature fo the vertex inhomogeneity of actual Nodaviridae would provide a decisive test of the model. Because current structural determination methods impose icosahedral symmetry, this is not yet possible, but we are hopeful that developments in these methods will make such a test feasible in the not-too-distant future.

We would like to thank Professors W. M. Gelbart and J. E. Johnson for helpful discussions. We are also grateful to Professor Johnson for permission to reproduce Fig. 1
[1] H. H. Bink and C. W. Pleij, Arch Virol 147, 2261 (2002).

[2] T. Odijk, Biophysical Journal 75, 1223 (1998).

[3] S. Riemer and V. A. Bloomfield, Biopolymers 17, 785 (1978).

[4] N. V. Hud, Biophysical Journal 69, 1355 (1995).

[5] R. Bundschuh and T. Hwa, Phys. Rev. E. 65, 031903/1 (2002).

[6] A. J. Fisher and J. E. Johnson, Nature 361, 176 (1993).

[7] T. S. Baker, N. H. Olson, and S. D. Fuller, Microbiol Mol Biol Rev 63, 862 (1999).

[8] J. A. Speir, S. Munshi, G. Wang, T. S. Baker, and J. E. Johnson, Structure 3, 63 (1995).

[9] H. Tsuruta, V. S. Reddy, W. R. Wikoff, and J. E. Johnson, J Mol Biol 284, 1439 (1998).

[10] B. V. Prasad, D. O. Matson, and A. W. Smith, J Mol Biol 240, 256 (1994), 94300601 0022-2836 Journal Article.

[11] T. S. Baker, R. H. Cheng, J. E. Johnson, N. H. Olson, G. J. Wang, and T. S. Schmidt, in Proc. Electron Microsc. Soc. Am. (San Francisco Press, San Francisco, 1992), vol. 50, pp. 454-455.

[12] B. Böttcher and R. A. Crowther, Structure 4, 387 (1996).

[13] L. Tang, K. N. Johnson, L. A. Ball, T. Lin, M. Yeager, and J. E. Johnson, Nature Structural Biology 8, 77 (2001).

[14] Tang, et all [13] suggest that the paradox might be resolved once we allow the RNA1 molecule to "mix" in with RNA2 as part of the dodecahedral cage. Although possible, this is less elegant, in view of the dominant role that RNA2 plays in viral self-assembly.

[15] B. Bollobás, Graph theory: an introductory course, Graduate texts in mathematics ; 63. (Springer Verlag, New York, 1990), corrected third printing. ed.

[16] The actual theorem states that an Euler path is impossible if there are more than two vertices connected to an odd number of edges. In both the dodecahedron and the icosahedron all vertices have an odd number of edges incident.

[17] S. K. Nechaev, Statistics of knots and entangled random walks (World Scientific, Singapore ; River Edge, NJ, 1996), s.K. Nechaev. Includes bibliographical references and index.

[18] C. W. Pleij, K. Rietveld, and L. Bosch, Nucleic Acids Res 13, 1717 (1985).

[19] An interesting historical footnote is that the notion of a Hamiltonian path originated in the construction by William Rowan Hamilton of a puzzle, called the "Icosian Game," the object of which is to find a Hamiltonian path along the vertices of a dodecahedron.

[20] S. J. Flint, Principles of virology : molecular biology, pathogenesis, and control (ASM Press, Washington, D.C., 2000).

[21] P. K. Sorger, P. G. Stockley, and S. C. Harrison, J Mol Biol 191, 639 (1986). 